FORMATION Formation emploi

Revue française de sciences sociales

149 | Janvier-Mars 2020

Varia

\title{
La professionnalisation des formateurs dans le cas d'un métier en devenir, celui d'ostéopathe
}

Vocational training for a recent profession, osteopathe

Die Professionalisierung der Ausbilder in einem neuem Berufs

La profesionalización de los formadores en el caso de una profesión en

construcción, la de osteópata

Dr. Vivien Braccini, Franck Garnier et Pr. Laurence Durat

\section{OpenEdition}

Journals

Édition électronique

URL : https://journals.openedition.org/formationemploi/8009

DOI : 10.4000/formationemploi.8009

ISSN : 2107-0946

Éditeur

La Documentation française

Édition imprimée

Date de publication : 31 mars 2020

Pagination : 87-111

ISSN : 0759-6340

Référence électronique

Dr. Vivien Braccini, Franck Garnier et Pr. Laurence Durat, « La professionnalisation des formateurs dans le cas d'un métier en devenir, celui d'ostéopathe », Formation emploi [En ligne], 149 | Janvier-Mars 2020, mis en ligne le 01 janvier 2022, consulté le 07 janvier 2022. URL : http://

journals.openedition.org/formationemploi/8009; DOI : https://doi.org/10.4000/formationemploi.8009

(c) Tous droits réservés 


\title{
La professionnalisation des formateurs dans le cas d'un métier en devenir, celui d'ostéopathe
}

\begin{abstract}
Dr. VIVIEN BRACCINI
Chargé de recherche chez PS International, entreprise de R\&D en sciences humaines et sociales, et chercheur associé au Laboratoire interuniversitaire des sciences de l'éducation et de la communication (LISEC) EA2310, équipe Activité, Travail et Identité Professionnelle (ATIP).

Docteur en sciences de l'éducation

Franck GARnier

Enseignant-chercheur au Collège ostéopathique Strasbourg Europe, et doctorant en sciences de l'éducation au Laboratoire interuniversitaire des sciences de l'éducation et de la communication (LISEC) EA2310, équipe Activité, Travail et Identité Professionnelle (ATIP).

Ostéopathe DO

Pr. Laurence Durat Enseignante-chercheuse au Laboratoire interuniversitaire des sciences de l'éducation et de la communication (LISEC) EA2310, équipe Activité, Travail et Identité Professionnelle (ATIP).

Professeure en sciences de l'éducation
\end{abstract}

Résumé

La professionnalilsation des formateurs dans le cas d'un métier en devenir, celui d'osthéopathe

La professionnalisation des formateurs constitue généralement l'une des étapes du processus de professionnalisation d'un métier. C'est pourquoi nous proposons d'interroger les enjeux et les conditions de l'élaboration d'un référentiel de compétences de formateurs d'un métier récent. C'est à partir des résultats de la phase exploratoire d'une recherche intervention, commencée il y a deux ans, que notre équipe explore le cas d'un important réseau français d'écoles d'ostéopathie, afin d'exposer sa compréhension des besoins de professionnalisation des formateurs ostéopathes.

Mots clés: métier de la santé, formateur, formation de formateur, professionnalisation, référentiel métier, établissement d'enseignement, enseignement privé, enseignement supérieur, ingénierie de la formation, identité professionnelle 


\section{Vocational training for a recent profession, osteopathe}

Vocational training is generally one of the stages in a professional training process for a given profession. This is why we propose to study the challenges and conditions for developing a skills benchmark for trainers for a recent profession. Using results from the exploratory phase of research carried out in a large French network of osteopathy schools, our team explores the network's understanding of the vocational training needs of their trainers.

Keywords: occupation in the health sector, trainer, trainer training, professionalisation, job benchmark description, educational institution, private education, higher education, training media, professional identity

Journal of Economic Literature: I 23, I 19

Traduction : auteur.e.s

\section{Introduction}

Malgré son siècle d'existence, l'ostéopathie n’a été légalisée, en France, que depuis 2002. Il faut attendre les décrets de 2007 pour spécifier son champ d'action par rapport à la médecine, et encadrer l'activité des nombreuses écoles privées apparues depuis. Et ce n'est qu'en 2014 qu'un référentiel de compétences est paru, inspiré par les travaux de Michel Tardif (2012), doublé d'un premier référentiel de formation. Cette évolution législative témoigne du processus, toujours en cours, de professionnalisation de cette discipline. La structuration de son système de formation est une composante logique de ce processus. Elle nous amène à interroger la nature des compétences des formateurs de la discipline pour laquelle aucun référentiel de formateur n'est disponible.

Afin d'améliorer la qualité de la formation des étudiants ostéopathes via la professionnalisation de leurs formateurs, mais aussi de mieux comprendre les conditions de transmission d'un métier de l'humain et du développement de son intelligence professionnelle, nous avons proposé une recherche intervention à un réseau d'écoles privées agréées.

Dans cet article, nous nous appuierons sur les résultats de la première étape de cette recherche intervention qui s'inscrit dans les sciences de l'éducation. Nous mobiliserons, à ce titre, le concept de "professionnalisation ", éclairé à la fois par le point de vue de la sociologie de la formation et des professions, et par des courants de l'analyse de l'activité. Grâce à cette approche multi-disciplinaire, nous interrogerons les liens entre l'institutionnalisation de la profession de formateur et celle du métier préparé. Les enjeux qui en découlent nous conduiront à proposer notre perception des besoins 
de professionnalisation du métier de formateur ostéopathe, et des conditions permettant d'y répondre, et ainsi de contribuer à la meilleure connaissance des processus de professionnalisation.

\section{La professionnalisation des métiers : le cas des formateurs ostéopathes}

Avant d'explorer le cas spécifique des formateurs ostéopathes, il apparaît pertinent de revenir sur la notion polysémique de professionnalisation et la place normative qu'y tiennent les référentiels. Nous pourrons alors souligner les problèmes que pose cette normativité dans le cas des métiers du soin manuel, et les difficultés du recours aux référentiels de formation dans le cas des formations initiales professionnalisantes.

\subsection{La professionnalisation, un processus en trois dimensions}

La professionnalisation d'un métier est un processus historique qui connaît quatre étapes : l'existence d'une pratique non reconnue par les instances juridiques et étatiques (1), une reconnaissance (2) visant la régulation économique et sociale de l'activité qui débouche sur une négociation collective (3) définissant ce que doit être le métier. Enfin, on observe une centralisation des pouvoirs (4) au sein d'instances chargées de réguler et contrôler les normes établies (Dubar \& Tripier, 1998). Ce processus, grâce auquel un groupe construit la reconnaissance des spécificités de sa profession, s'opère à travers l'évolution des interactions entre les individus, les institutions et la société. C'est pourquoi Richard Wittorski (2009) distingue, sous ce même vocable, trois phénomènes différents, mais complémentaires : la «dynamique d'élaboration » d'une profession portée par les groupes sociaux, la " mise en mouvement des individus » portée par les organisations visant la flexibilité et l'adaptabilité, et la « fabrication » de professionnels légitimes qui est la raison d'être des institutions de formation. Pour Wittorski (2015), la professionnalisation fait donc interagir la définition identitaire de référence du collectif de travail, le développement professionnel des individus et la dynamique des négociations identitaires entre référentiels institués et activités singulières situées. Ces trois dimensions de la professionnalisation peuvent s'appuyer sur un référentiel de compétences (RC) dont le rôle est de rendre possible l'évaluation des écarts entre une norme socialement stabilisée et l'activité humaine. L'implication des partenaires sociaux et du gouvernement dans sa création constitue une partie de la « dynamique d'élaboration ". S’il est également vu comme un outil formatif (Parent, 2008) faisant l'objet d'un travail continu et collectif d'élaboration et d'actualisation par les usagers du référentiel eux-mêmes, le RC soutient alors " la mise en mouvement des personnes ". Enfin, en servant à la production et à l'évolution des référentiels de formation, le RC favorise la «fabrication " des professionnels du champ. 


\subsection{Risques de la rationalisation des métiers du soin manuel}

Le recours au référentiel peut être très utile à la professionnalisation. Mais il existe un risque de domination de la logique de contrôle et d'efficacité portée par la vision technocratique. Le référentiel devient alors un outil de contrôle de la conformité, et le travail est réduit à une forme figée et atomisée incompatible avec le caractère indivisible, complexe et dynamique des situations de travail (Pastré, 1997), sur lequel se construit la compétence (Durat \& Mohib, 2008). L'impossibilité de négocier et de faire évoluer la norme limite la négociation identitaire et le potentiel apprenant des situations de travail (Mayen, 2018).

Ces contraintes sont exacerbées dans le cas des métiers relationnels où le facteur humain tient une place importante, car la qualité du travail dépend de l'adaptabilité, de l'éthique, et des choix de posture professionnelle. Or, ces éléments reposent sur des conceptions du rôle et des fonctions des acteurs de la relation (Wittorski, 2015). La remarquable singularité qui s'en dégage rend difficiles à la fois l'explicitation des structures de l'activité et la transmission du métier. Les professions du soin manuel ne sont pas épargnées. Comme le souligne Balas (2011) dans le cas des kinésithérapeutes, "la réussite de l'acte [de soin] passe par une forme de "coproduction" en partie inconsciente, où le patient joue aussi un rôle majeur". Cette part subjective du travail (Desjours, 2001) est encore renforcée par l'importance des compétences corporelles et sensorielles, telles que " la sensibilité palpatoire » et la perception des teintes ou des sons du corps qui s'acquièrent avec la pratique. Comme pour les kinésithérapeutes, c'est "avec l'expérience, [que] ces professionnels découvrent, de manière empirique, des manières de faire à l'efficacité éprouvée plutôt que prouvée" (Balas, 2012).

\subsection{Référentialisation et formation professionnalisante}

Pour former les futurs acteurs du soin, les disciplines de soin parallèles s'appuient sur des écoles à finalité professionnalisante (Wittorski, 2015) qui visent donc l'acquisition de savoirs évalués par des critères de légitimité du métier, quand les formations professionnelles portent sur des savoirs d'action évalués par des critères d'efficacité in situ. L'intérêt des formations professionnalisantes est de soutenir la création d'une identité professionnelle en reliant les contenus enseignés à l'activité professionnelle, tout en permettant un degré d'adaptation (Parent \& Jouquant, 2015). Elle apparaissent donc essentielles et complémentaires à la formation professionnelle lorsque le potentiel d'adaptation devient insuffisant, dans le sens où la formation professionnelle est plus difficile à recevoir si les individus ne possèdent pas les normes partagées du métier. Mais cette normalisation introduite par la référentialisation des formations ne permet pas d'homogénéiser pleinement la formation. Son caractère non situé oblige les formateurs à interpréter les compétences qui y figurent "selon leur propre cursus scolaire et leur trajectoire professionnelle" (Agulhon, dans Bourdoncle \& Demailly, 1998). 
Ainsi, il existe une inévitable variabilité des formateurs, commune à toute formation professionnalisante. Mais certaines origines de cette variabilité sont propres à l'ostéopathie, ce qui nous amène à présenter brièvement ce métier et son système de formation.

\section{Le cas de l'ostéopathie et son contexte}

Après un court rappel de ce qu'est cette pratique de soin, nous décrirons le dispositif de formation afin d'exposer les enjeux socio-économiques actuels de ce secteur en plein développement, et ce afin de mettre en évidence la question du recrutement des formateurs.

\subsection{L'ostéopathie, de quoi parle-t-on ?}

Selon Issartel \& Issartel (1983), l'ostéopathie consiste à agir sur les déséquilibres de la structure corporelle limitant ou bloquant la circulation de l'information et des substances nécessaires au fonctionnement du corps, à l'origine de réactions compensatrices. Ces réactions sont chargées d'assurer la continuité des fonctions de l'organisme, mais occasionnent des troubles chez l'individu (douleurs, perte de mobilité, etc.). En agissant sur les déséquilibres, le thérapeute fait disparaître ces compensations pour donner au corps l'opportunité de rétablir son fonctionnement. Cette définition s'appuie sur divers principes tels que l'unité du corps, l'interdépendance entre la structure et la fonction des organes ou l'auto-guérison du corps (Issartel \& Issartel 1983 ; Coues, Hoffman \& Comeaux, 2012). Ces principes découlent du " concept ostéopathique " qui est une manière de considérer la santé humaine à travers un prisme écologique prônant l'adéquation entre les individus et leur environnement, et qui rejoint en cela la conception de l'Organisation mondiale de la santé (OMS). Celle-ci a défini la santé comme étant "un état de complet bien-être physique, mental et social, et qui ne consiste pas seulement en une absence de maladie ou d'infirmité. " Pour agir, l'ostéopathe s'appuie sur un ou plusieurs de ces principes fondamentaux qui justifient le recours à certaines pratiques et techniques de soin plutôt qu'à d'autres. L'approche globale, systémique et interactionnelle de l'ostéopathie, explique l'existence de plusieurs modalités d'intervention pour une même situation de soin. Prenons le cas d'une perte de mobilité douloureuse à l'épaule. Le thérapeute peut agir sur les tissus conjonctifs de l'épaule qui relient les composantes du corps (muscles, os, tendons, etc.). Il peut également intervenir sur des viscères, en l'occurrence le foie, car il

1. Premier principe de la constitution de l'Organisation mondiale de la santé (OMS), page 1. En ligne : https://www.who.int/fr/about/who-we-are/constitution 
semble exister une causalité empirique entre les spasmes du foie et les douleurs d'épaules. Ces deux approches visent le même but : agir sur le système nerveux et vasculaire. Le thérapeute élabore ainsi son intervention en fonction de la situation du patient, mais aussi de son interprétation du "concept ostéopathique " qui est au fondement de son "modèle opératif» (Vidal-Gomel \& Rogalski, 2007).

\subsection{La formation professionnalisante des écoles d'ostéopathie}

Depuis les décrets de 2014, l'étudiant ostéopathe se prépare à son métier en suivant une formation de 4860 heures, répartie sur cinq années. Ce cursus prévoit 1546 heures de cours magistraux et 1814 heures de travaux dirigés. S'ajoutent 1500 heures de formation pratique avec patients, dont les deux tiers au moins sont à réaliser en clinique pédagogique. Parmi ces heures, 300 environ sont consacrées à la réalisation obligatoire de 150 consultations " complètes, validées " par un ostéopathe DO (diplôme d'osthéopathe). Les apports théoriques se concentrent sur les trois premières années et se réduisent progressivement au profit de la formation pratique.

Lors des cours magistraux, le taux d'encadrement n'est pas spécifié, tandis que lors des travaux dirigés ou pratiques, le taux d'encadrement doit être d'un formateur pour 35 étudiants. Cette formation peut être allégée grâce à des équivalences passées dans d'autres formations médicales ou paramédicales. Avec cette réforme, les médecins et autres métiers de santé ne peuvent plus obtenir le diplôme d'ostéopathe à partir d'une simple spécialisation ou de stages, car l'ensemble des heures de pratiques sont devenues obligatoires, soit deux à trois années de formation à temps plein selon le cas.

\subsection{Les enjeux socio-économiques de la référentialisation}

Malgré les avancées législatives et la structuration de la formation, le Figaro du 3 février 2017 publiait "Les écoles d'ostéopathie épinglées pour leur manque de sérieux ». Le président du syndicat français des ostéopathes (SFDO) y précise que sur la trentaine d'écoles reconnues, "on va de l'excellence à la médiocrité ". Ce constat est sans doute lié au fait qu'une partie de ces écoles conservent leur agrément ministériel, malgré une application relative de la nouvelle législation. Le retrait de l'agrément semble créer des difficultés de gestion des étudiants en cours de cursus. Que faire pour ces étudiants ayant obtenu un diplôme invalide leur ayant coûté plusieurs dizaines de milliers d'euros (la formation étant payante et non subventionnée) ?

En réaction à ce constat et pour mieux se différencier sur un marché concurrentiel, une partie des écoles françaises ont obtenu l'inscription de leur diplôme au Répertoire national des certifications professionnelles (RNCP). Cette initiative n'est pas sans rappeler le processus d'institutionnalisation de la médecine chinoise étudié, par Delphine Parent (2017). L'auteure décrit une concurrence entre les organisations syndicales, les associations professionnelles ou les groupes d'écoles pour établir la déon- 
tologie et les normes de qualité et de sécurité de référence. Elle montre ainsi que la reconnaissance de la discipline passe par l'exclusion d'une partie de la communauté professionnelle.

Au moins deux courants d'écoles d'ostéopathie française émergent de cette concurrence. Le premier courant tente d'appuyer leur développement sur l'institutionnalisation de la discipline et le rehaussement de la qualité des formations qui repose, entre autres, sur le respect du référentiel de formation. Mais un second courant a choisi d'alléger les contraintes en ne respectant que partiellement les nouveaux cadres de références, en ne respectant pas les volumes horaires du référentiel formation ou le taux d'encadrement. Il est possible qu'à l'instar d'autres branches de soin parallèles, comme la médecine chinoise (Parent 2017), une part des écoles s'oppose à une normalisation étatique du métier et de sa formation, en revendiquant une liberté de conception et de pratique. Mais il est encore plus probable que la rentabilité d'une partie des écoles soit remise en cause du fait de leur trop petite taille pour amortir les surcoûts de fonctionnement occasionnés par la nouvelle législation.

En effet, prenons l'exemple du volume horaire consacré à la clinique pédagogique. Les écoles, en doublant ce volume horaire, doivent souvent acquérir de nouveaux locaux et financer des heures d'encadrement supplémentaires. De même, la règlementation qui impose un formateur pour vingt-cinq apprenants, tant pour les travaux pratiques que dirigés, nécessite des embauches. Certains y verront d'ailleurs une stratégie étatique visant la fermeture des écoles jugées dangereuses pour les patients des futurs praticiens mal formés, ou de celles qui fixent des coûts très élevés, sans expliciter les risques de pertes financières pour les apprentis ostéopathes, voire d'endettement, en raison de la saturation du marché de l'ostéopathie qui ne garantit pas à tous les nouveaux praticiens des revenus suffisants pour amortir le coût de leur formation.

\subsection{Recrutement de professionnels et formation sur le tas}

Dans ce contexte, le recrutement et la compétence des formateurs deviennent un enjeu stratégique et expliquent l'acceptation de notre projet de recherche. Actuellement, la professionnalisation des formateurs en ostéopathie tend à suivre une logique de formation sur le tas qui consiste à recruter des professionnels de la discipline enseignée et à compter sur la pratique pour former à l'enseignement. Les compétences de thérapeute sont incontournables, car elles garantissent la maîtrise technique et le respect des usages. Mais cette logique ne garantit pas la qualité et l'efficacité d'une formation, ne serait-ce que parce qu'elle n'assure pas la constitution d'une identité professionnelle propre au groupe des formateurs en ostéopathie, ces formateurs risquant de s'identifier avant tout à leur activité de thérapeute avant celle de pédagogue. Précisons d'ailleurs que si nous employons le terme de formateur, on constate l'emploi indifférencié des termes de formateur et d'enseignant au sein des écoles, alors que le public encadré est composé de jeunes adultes, que la finalité de la formation est professionnalisante et que l'anticipa- 
tion de l'usage des apprentissages est élevée (Boutinet, 1995). C’est la raison pour laquelle nous utiliserons les termes de formateurs et de formés, même si, comme nous le verrons, ce clivage n'est pas si marqué et interpelle justement la question de l'identité professionnelle. Rappelons que l'identité professionnelle est un construit, un état en devenir qui s'élabore dans les interactions (Fray \& Picouleau 2010) où se joue la reconnaissance de soi par soi, par les pairs et par une communauté d'appartenance. Cette reconnaissance s'appuie sur une culture propre aux équipes, aux entreprises ou aux groupes professionnels, qui permet de se distinguer et de se définir par rapport à l'extérieur. Cette culture s'exprime à travers l'apparition d'objets, de croyances, de normes et de comportements communs (Liu, 2013). Elle génère un sentiment d'appartenance qui étaye l'engagement des personnes dans leur développement professionnel.

La professionnalisation des formateurs à une discipline naissante ne soulève pas que des questions didactiques et pédagogiques. Elle amène aussi des questions socio-économiques qui interpellent ses liens avec les modalités de structuration du métier lui-même.

\section{Quand la fragilité de l'identité d'une profession et de ses formateurs semble nuire à l'apprentissage des formés}

Malgré cette normalisation législative, nous avons observé, de façon récurrente, des résultats inégaux aux examens, au sein de groupes d'étudiants d'une même école, et ce, pour des fonctionnements et des profils d'étudiants proches. Nous avons supposé que cette variabilité est "formateur-dépendante » (Garnier \& Braccini 2017), car Agulhon (1998) montre que l'enseignement est une vraie profession, et que les formateurs sont rarement diplômés en pédagogie. Il paraissait donc plausible qu'il existe un déficit de compétences en pédagogie qui, par la nature des techniques de formation utilisées et la nature des relations établies, limiterait la capacité cognitive et conative des formés à apprendre et à réussir aux examens.

Mais cette variabilité de qualité d'enseignement, suggérée par les résultats aux examens, peut également s'expliquer par une différence d'interprétation du référentiel de compétences tenant à la double singularité qui caractérise les métiers du soin manuel : celle liée à la relation humaine, et celle relative aux dimensions palpatoires de la discipline. Comme nous l'avons vu, cette double singularité rend la transmission de ces métiers difficiles, et oriente la compréhension de leur référentiel selon l'expérience du formateur. Ainsi, s'il apparaît opportun de professionnaliser ce métier de formation pour répondre aux enjeux de la professionnalisation de l'ostéopathie, une question essentielle s'impose : pour développer et évaluer les compétences des professionnels de ces écoles, quelles définitions de l'ostéopathe et du formateur ostéopathe utiliser comme référence, connaissant leur intrication? 
L'établissement de ces références suppose de cibler les éléments significatifs et partagés par la majorité des instances représentatives de la profession ${ }^{2}$ et des écoles, concernant les définitions, les usages et le champ d'application de la discipline. En effet, la vision jugée légitime détermine les objectifs de la formation, et donc les compétences des formateurs nécessaires à leur atteinte. Or, dans le cas étudié, la diversité des approches de soin et des pratiques nous amène à penser que l'image du "bon thérapeute " n'est justement pas partagée. Aussi est-il possible, par extension, que ce déficit identitaire de la profession engendre un déficit identitaire chez les formateurs, ce qui contribue à augmenter les difficultés d'apprentissage du métier chez les étudiants. En effet, utiliser de manière isomorphique l'expérience scolaire, comme on le rencontre souvent chez les enseignants (Poteaux, 2009), n'est pas adapté à la finalité professionnalisante de la formation et à ses ingénieries (Ardoin, 2015).

Pour faire évoluer la qualité de l'action des formateurs à partir de l'évolution de leur identité professionnelle, une méthodologie particulière s'est avérée nécessaire.

\section{Méthodologie : une recherche-intervention en quatre étapes}

Pour favoriser une bonne compréhension de notre méthodologie, nous proposons de restituer d'abord la démarche globale de notre recherche-intervention, afin de situer la phase exploratoire utilisée dans cet article et de détailler l'échantillon du questionnaire qui y tient un rôle important.

\subsection{Design global de la recherche-intervention}

Notre recherche se rapproche de la recherche-intervention, même si elle ne s'en réclame pas explicitement, puisque l'on y retrouve simultanément des finalités de compréhension, de transformation de la situation étudiée et de formation des participants. Les acteurs sont impliqués dans la production, l'interprétation ou la validation des données (SaintJean \& al., 2014). Néanmoins, les chercheurs restent maîtres des méthodes et outils d'investigation, ainsi que du cadrage théorique (David, 1999).

Cette recherche s'opère en quatre étapes : une exploration du terrain pour évaluer la situation des formateurs ostéopathes (objet de cet article) ; l'identification et l'enregistrement de situations de formation pertinentes au regard des questions tirées de l'exploration ;

2. Notamment l'Union pour l'ostéopathie qui est une fédération de syndicats. On y retrouve le Syndicat français des ostéopathes (SFDO), le Syndicat national des ostéopathes du sport (SNOS), la Fédération nationale de l'enseignement supérieur en ostéopathie (FNESO), ainsi que la Fédération nationale des étudiants en ostéopathie (FEDEO). 
l'exploitation des traces à partir d'une méthode d'analyse collective de l'activité ; et enfin, la production de recommandations. Pour mener cette démarche, notre équipe se compose de trois chercheurs en sciences de l'éducation.

\subsection{Méthodologie de la phase exploratoire, première étape de la recherche}

Faute de littérature sur le métier, nous avons décidé d'explorer le terrain à partir d'une démarche clinique qui se fonde sur une triangulation des sources (De Ketele \& Maroy, 2006). Ainsi, les indices servant à notre raisonnement orienté par notre immersion, sont issus d'entretiens individuels, d'entretiens collectifs d'approfondissement et d'un questionnaire de généralisation en ligne.

Les entretiens individuels semi-directifs sont au nombre de six. Leur retranscription et leur analyse ont fait l'objet d'une publication (Garnier \& Braccini, 2017) à laquelle nous renvoyons le lecteur pour plus de détails. Ils ont été menés, en 2016, dans une première école de la région Grand Est, auprès de formateurs volontaires, tous ostéopathes diplômés. L'un d'eux était médecin, un autre kinésithérapeute et un troisième manager. Ils étaient âgés de 37 à 57 ans et avaient entre cinq et trente ans d'expérience de formation en ostéopathie. Le guide d'entretien avait été élaboré à partir d'une analyse réflexive de l'expérience d'un membre de notre équipe, ancien coordinateur pédagogique. Ces entretiens nous ont permis d'identifier divers thèmes et hypothèses concernant la définition des métiers d'ostéopathe et de formateur-ostéopathe.

Nous avons approfondi ces résultats à l'aide de trois entretiens collectifs d'une durée de deux à trois heures chacun, menés fin 2017, dans une seconde école du Grand Est. Ils impliquaient une dizaine de formateurs, dont quatre étaient présents à chaque rencontre. Les participants étaient majoritairement des jeunes formateurs et praticiens, avec moins de dix ans d'expérience. Les guides d'entretien se fondaient sur les résultats des entretiens individuels de 2016, et sur les matériaux dégagés d'un entretien à l'autre. Ces entretiens enregistrés ont permis de retranscrire et réorganiser les thématiques et les principales " unités de sens" (Bardin, 2013), sous la forme de carte mentale à partir de laquelle nous avons rédigé les synthèses.

Pour conforter nos résultats et interprétations, les six écoles du réseau commanditaire, réparties sur l'ensemble du territoire français, ont été destinataires, en 2018, d'un questionnaire réalisé à partir du logiciel Sphinx Déclic. Le tri à plat des données a été complété par des tableaux croisés dynamiques, et des analyses factorielles des correspondances multiples (Escofier \& Pagès, 2008). Celles-ci ont été écartées car les signes d'interdépendance entre variables, ou de clivages dans les projections, étaient trop faibles, notamment celles impliquant l'ancienneté, l'âge et l'origine géographique. 


\subsection{L'échantillon de formateurs pour le questionnaire écrit}

Le réseau commanditaire regroupe environs 120 formateurs ostéopathes. Après stabilisation du nombre de réponses, l'échantillon aléatoire obtenu s'est composé de soixanteseize sondés, soit $62 \%$ de l'échantillon parent. Les formateurs destinataires sont tous diplômés en ostéopathie et cumulent une activité d'ostéopathe, sans que nous en connaissions toutefois le volume horaire. Pour les six écoles, près de $30 \%$ sont des femmes, $26 \%$ ont plus de 40 ans et $38 \%$ ont moins de 32 ans. L'âge moyen des hommes est de 38 ans, contre 34 pour les femmes. Le sexe, l'âge ou l'ancienneté n'ont pas permis d'observer une répartition des réponses propres aux écoles.

L'échantillon comporte peu d'ostéopathes reconvertis, puisque une dizaine d'entre eux seulement possède une formation antérieure dans le milieu de la santé, dont un médecin et sept kinésithérapeutes. Une dizaine d'autres personnes possède des diplômes d'université (DU) servant de spécialisation à l'ostéopathie, et une autre dizaine affiche des diplômes de type licence, maîtrise, doctorat, faiblement reliés au métier, mais qui ne semblent pas avoir donné lieu à une première carrière, si l'on tient compte de l'âge des personnes. Ce constat s'explique par l'obligation d'être diplômé d'État pour prétendre à la fonction de formateur. Tous les ostéopathes formés avant la reconnaissance du métier, en 2007, première phase de professionnalisation (Dubar \& Tripier, op. cit.) de l'ostéopathie, sont écartés des écoles, faute de diplôme d'État.

Notre échantillon de formateurs est donc assez homogène car les variables du profil des répondants n'apparaissent pas comme des facteurs clivants dans la répartition des réponses. Malgré tout, nous restons prudents quant à nos interprétations et nos conclusions, car la faible taille et la représentativité partielle de l'échantillon réduisent le choix des méthodes de traitement de données, nous cantonnant ainsi aux techniques descriptives et exploratoires à visée interprétative.

Nous allons maintenant exposer les matériaux obtenus avec cette méthodologie et les interprétations réalisées à partir de notre cadre théorique afin de cerner les représentations des formateurs sur leur activité de thérapeute et de formateur.

\section{Des représentations de l'ostéopathe non prises en compte dans la formation et une vision scolaire de celle-ci}

Pour parvenir à formuler des recommandations susceptibles d'améliorer les compétences des formateurs et l'apprentissage des formés, nous avions besoin de connaître les représentations et les perceptions qui ont cours chez les actuels formateurs. Nous montrerons que la dimension humaine du métier est bien perçue, même si elle est faiblement prise en compte dans la formation. Toutefois, cette vision commune n'empêche pas un certain éclectisme dans la définition de l'ostéopathe compétent. 
Ce point, qui ne favorise pas l'homogénéisation de l'action de formation au sein des écoles, s'ajoute aux effets du profil des formateurs, dont la tendance est d'être peu expérimentés et peu diplômés en formation des adultes. Ces éléments se trouvent renforcés par l'observation de courants au sein des formateurs ayant une conception plutôt relationnelle ou technique de l'ostéopathe. Enfin, quels que soient ces courants, la vision du métier de formateur est très largement marquée par le modèle scolaire faiblement adapté à la logique de professionnalisation, étayant ainsi encore davantage la nécessité de professionnalisation des formateurs ostéopathes au métier de la formation des adultes.

\subsection{L'ostéopathe compétent est humain, donc efficace ?}

D'après les entretiens collectifs d'avant enquête, la définition d'un ostéopathe compétent nous rapproche de la dimension efficacité de la notion de compétence (Durat $\&$ Mohib, 2008). Il doit être un thérapeute capable d'apporter un soulagement à son patient après son intervention. Deux groupes essentiels de compétences apparaissent nécessaires pour cela : celui des compétences techniques de soin et celui des compétences relationnelles, confirmant ainsi l'appartenance du métier à ceux de l'humain (Bordergrat \& Buznic-Bougeacq, 2015). Le second groupe de compétences vise la compréhension de la personne, de sa situation et de son besoin. Il se décline par les notions d'écoute et d'empathie qui visent à placer le patient dans des dispositions psychologiques, physiques et affectives propices au soin. Toutefois, pour être relationnellement compétent, l'ostéopathe se doit aussi d'être pédagogue, afin de faire comprendre au patient la réinterprétation de sa situation du point de vue de l'ostéopathie et l'action qui va en découler. À la pédagogie et à l'écoute, les professionnels interrogés ajoutent l'humilité, pour ne pas risquer de se substituer au patient dans la définition du besoin, ou douter de sa capacité à comprendre le soin. Ces deux groupes de compétences visent l'établissement d'une confiance réciproque suffisante pour que le patient se laisse manipuler et participe au soin (Balas, op. cit.).

\subsection{Une définition du métier d'ostéopathe faiblement partagée}

Si le graphique 1 souligne un partage relatif de cette définition, il met en évidence deux visions de la profession, l'une plutôt technicienne, tandis que l'autre est plutôt relationnelle. 


\section{Graphique 1. Classement des compétences clés du bon ostéopathe}

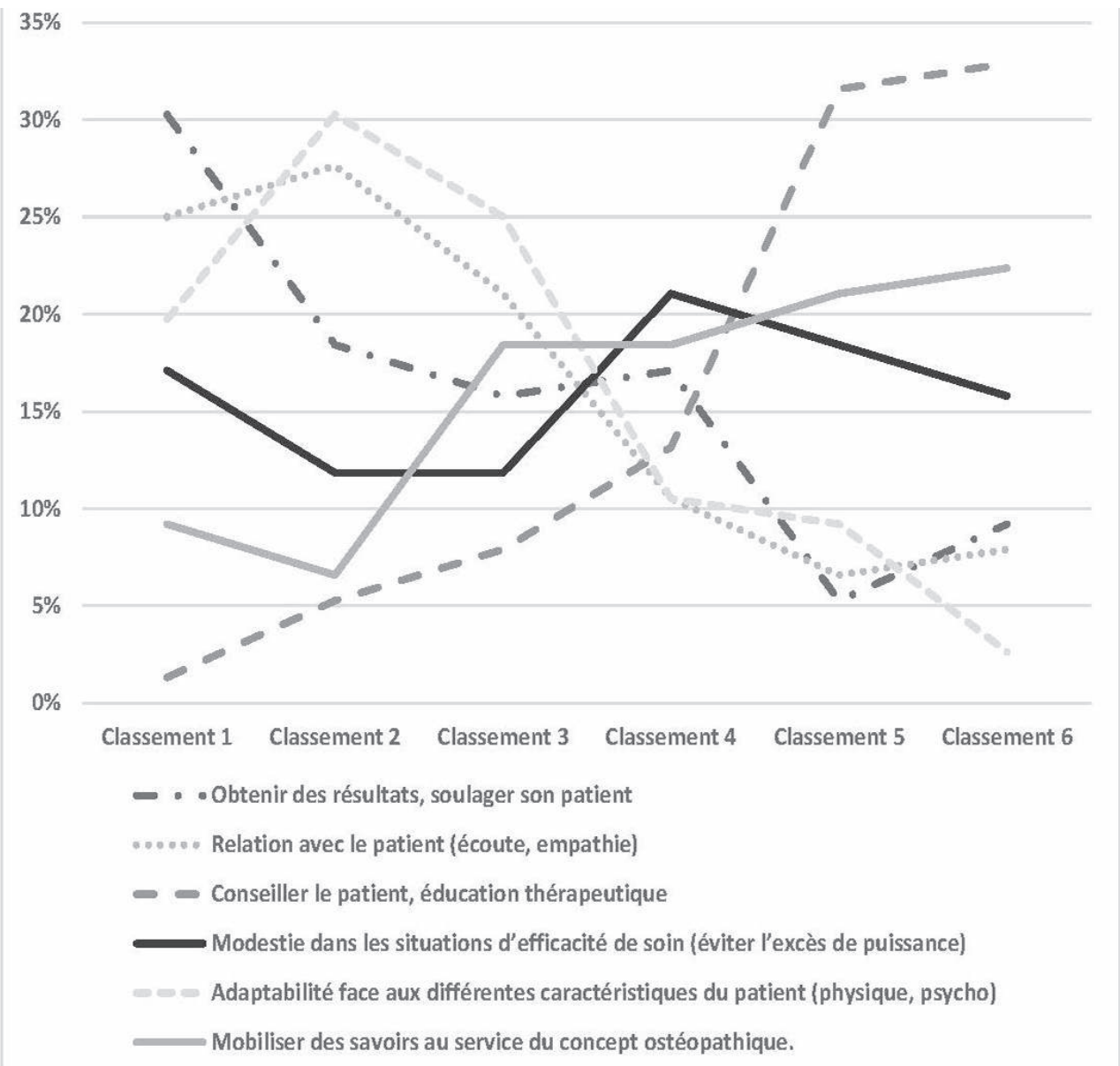

Note de lecture : $30 \%$ des sondés classent en seconde position d'importance l'adaptabilité face aux différentes caractéristiques du patient (physiques et psychologiques).

Source : Enquêtes sur les représentations et les pratiques des formateurs ostéopathes du réseau Franc'osteo.

Le graphique 1 est à double entrée : la somme des effectifs d'un même classement représente $100 \%$ de l'échantillon, tout comme la somme des différents classements d'une même variable. Ainsi, 30 \% de l'échantillon ont placé « l'obtention de résultats " en classement 1 , tandis qu'un peu moins de $10 \%$ ont choisi la modalité " mobiliser des savoirs au service du concept ostéopathique». 
Ainsi, le graphique 1 nous montre que même si les idées d'efficacité et de relation au patient sont majoritaires dans le premier et le second classement, leur cumul ne représente que $50 \%$, à côté de la modalité « l'adaptabilité aux caractéristiques du patient ». À l'inverse, la modalité « conseiller le patient et éducation thérapeutique » est jugée comme la moins importante, puisque classée, dans $65 \%$ des cas, dans les deux dernières positions. Il semble que la perception dominante du métier soit tournée vers l'intervention thérapeutique plutôt que vers la prévention, alors que l'approche globale du patient et la pérennité du soin font référence dans la littérature ostéopathique (Gueullette op cit., Coues \& al., op. cit. ; Le Corre \& Toffalani, op. cit. ; Issartel \& Issartel, op. cit.).

Cette répartition n'apparaît pas corrélée avec les autres variables évaluées. Les professionnels, bien qu'intégrant des éléments communs, ne les priorisent pas de la même façon. Cependant, les modalités liées à l'obtention des résultats, à la relation au patient et à l'adaptabilité semblent constituer la colonne vertébrale du métier, puisque présentes dans les trois premiers rangs dans plus de $65 \%$ des cas. La bonne répartition de la modalité " mobiliser des savoirs au service du concept ostéopathique " entre les différents classements relativise l'importance du "concept ostéopathique " évoquée. Pourtant, sa transmission est associée aux missions des écoles pour $40 \%$ des réponses, à égalité avec la transmission des techniques. Mais si faire élaborer le concept ostéopathique n'est pas une compétence clé, pourquoi sa transmission estelle une finalité importante des écoles ? Et qui, à part les formateurs, aurait le rôle d'en assurer l'acquisition ? Comment cette acquisition s'opère-t-elle actuellement ? Notre enquête met en évidence une certaine disjonction entre un discours qui axe la formation, le métier et les écoles sur la dimension technique, tout en affirmant les dimensions respectivement éthique et relationnelle indispensables à l'approche holistique du patient, qui ne sont actuellement pas évaluées dans le cursus. Cette disjonction pourrait être l'expression de la dérive de la référentialisation du métier, évoquée par Catherine Agulhon (1998), avec une domination d'une "professionnalisation-efficacité au travail " favorable à une approche médicale, au détriment d'une "professionnalisation-développement" plus en accord avec les principes spirituels de la discipline qui relient le bien-être de la personne à la quête de sens des individus, ainsi qu'à leurs relations sociales et environnementales. Nous observons, par ailleurs, que $88 \%$ des sondés considèrent la compréhension du patient comme étape clef de la consultation ostéopathique, alors que celle-ci repose sur ces dimensions relationnelles et éthiques sans laquelle l'anamnèse du patient est difficile. Cette confusion dans la définition du métier est l'expression des débats encore en cours. Elle révèle que l'ostéopathie n'a pas dépassé la négociation collective sensée stabiliser la définition du métier, troisième stade de sa professionnalisation (Dubar \& Tripier, op. cit.). 


\subsection{Le profil des formateurs à l'origine des disparités des performances des formés?}

Notre échantillon comporte une majorité d'hommes, avec 52 formateurs sur les 76 sondés, et sa population est plutôt jeune, avec $74 \%$ de moins de 40 ans et $38 \%$ de moins de 32 ans. L'âge peut être corrélé avec l'expérience professionnelle qui, dans $72 \%$ des cas, est inférieure à dix ans. Ces caractéristiques étayent l'hypothèse du rôle des formateurs dans le problème de disparité des performances des groupes d'étudiants. En effet, le manque d'expérience et la jeunesse des personnes, qui limitent les possibilités de " développement professionnel ", confèrent une place importante aux qualités individuelles et expliquent l'hétérogénéité des pratiques. Le manque pressenti de formation en pédagogie et didactique reste une autre explication possible. Avec seulement $15 \%$ de l'échantillon diplômé dans le champ de l'éducation et de la formation, l'absence de normes partagées, parmi les formateurs-ostéopathes, est inévitable, surtout dans le cas de la transmission des techniques qui repose en partie sur des compétences subjectives et incorporées (Desjours, op. cit.). Cela limite la négociation identitaire ${ }^{3}$ (Wittorski, 2009) et renforce notre hypothèse d'isomorphisme éducatif (Poteaux, 2009). Ce faible taux de diplômés fait également écho à la motivation à former, puisque $86 \%$ des interrogés le font d'abord pour transmettre la discipline, bien avant tout autre motif. On retrouve là un "attachement " à la discipline comparable à celui des enseignants du secondaire (Berger \& D’Ascoli, 2011), ce qui témoigne, selon nous, d'un ancrage dans le paradigme de la transmission plutôt que de l'apprentissage. Or, l'étude de la pédagogie dans le cadre d'un diplôme conduit à prendre du recul vis-à-vis de la discipline pour remettre l'apprenant au centre de l'activité de formation.

\subsection{Ostéopathe et technicien avant tout}

Pour les sondés, l'activité de formation constitue plus qu'une activité complémentaire à leur activité thérapeutique. Il s'agit bien d'une profession à part entière puisque $46 \%$ des formateurs déclarent former entre 100 et 300 heures par an, et $35 \%$ ont une activité supérieure à 300 heures annuelles. Rappelons qu'un service universitaire plein comporte 384 heures. Les formateurs ostéopathes cumulent donc deux métiers : ostéopathe et formateur ostéopathe, ce qui renforce la possibilité de l'influence d'un trouble identitaire du premier métier sur le second.

3. Les négociations identitaires portent sur les inévitables écarts entre les référentiels institués et les activités réelles qui sont situées et par là-même partiellement singulières.

4. Qui consiste à éduquer ou former autrui en reproduisant, consciemment ou non, la manière dont on a été soi-même éduqué et formé. 
Par ailleurs, la vision de l'ostéopathe apparaît avant tout axée sur la technicité. $44 \%$ des personnes de l'échantillon estiment que leur rôle principal de formateur est de faire des formés des professionnels fiables qui respectent la législation et la sécurité des futurs patients. Seul un quart environ des personnes de l'échantillon pensent qu'il est de leur ressort d'accompagner le cursus des individus et moins d'un quart que leur mission est de transmettre la philosophie de l'ostéopathie. Cette technicité conforte la perception du rôle principal des écoles qui, pour $40 \%$, est de former des techniciens ostéopathes. Pourtant, attribuer aux écoles le rôle de promoteur du concept ostéopathique confirme que la compétence de cette discipline n'est pas que technique, mais aussi humaine, dans la perspective d'un "patient-partenaire " commune aux métiers de la santé (Parent \& Jouquan, 2013).

Si notre enquête fait apparaître deux approches de l'ostéopathie, l'une plutôt technique et l'autre relationnelle, l'appartenance à ces approches repose certainement sur des caractéristiques intrinsèques. Aucun des croisements testés entre les résultats de la question portant sur les compétences et les variables du profil des répondants n'a fait apparaître de liens. Ces différences de conception pourraient provenir d'un positionnement épistémologique personnel vis-à-vis de la médecine, contre laquelle l'ostéopathie s'est historiquement construite (Gueullette, 2014). Comme dans le cas de la médecine chinoise (Parent, 2014), on retrouve un conflit entre une vision technique rationnelle héritée de la médecine expérimentale et une vision sensible spirituelle empruntant une partie de sa conception de l'humain et de sa psyché aux champs philosophiques, et parfois religieux.

\subsection{Former, c'est transmettre et faire comprendre}

Intéressons-nous à présent aux représentations du métier de formateur. Le graphique 2, synthétisant les représentations du "bon formateur ostéopathe " tend à révéler la prévalence d'une vision " enseignante " du métier, confirmant ainsi notre hypothèse portant sur le paradigme éducatif des formateurs, plutôt centrés sur la transmission de contenus, fondé sur leur expérience scolaire passé. Dans cette question à choix multiples, la somme des effectifs dépasse les $100 \%$.

Le graphique 2 permet uniquement de dire si la modalité fait partie de ce choix multiple, mais pas d'indiquer la position occupée dans ce choix. Ainsi, dans $58 \%$ des cas, la modalité "susciter l'intérêt de la matière " fait partie des trois principales compétences du formateur. 


\section{Graphique 2. Les trois principales compétences du formateur ostéopathe}

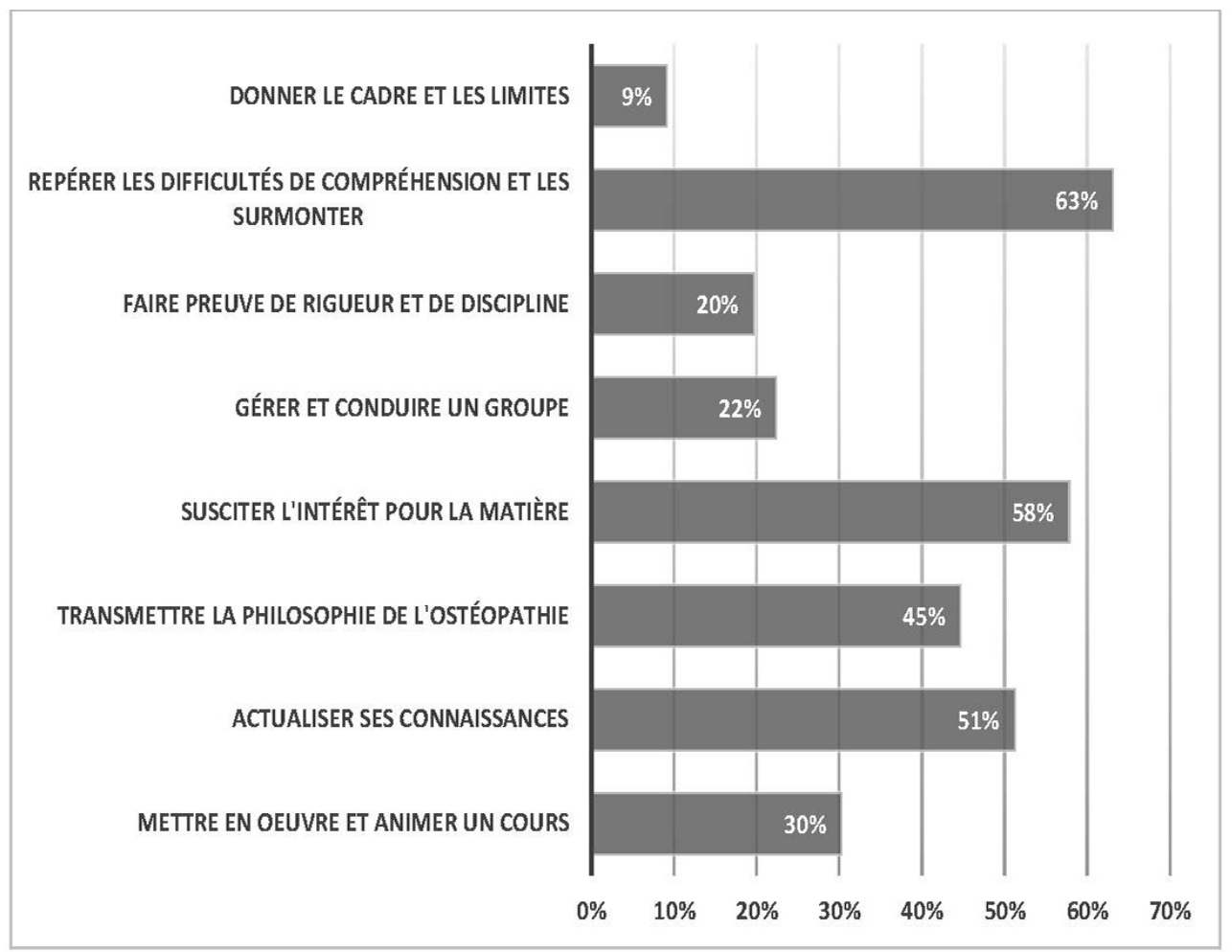

Note de lecture: $57 \%$ des sondés considèrent que « susciter l'intérêt de la matière » fait partie des trois compétences les plus importantes d'un formateur en ostéopathie.

Source : Enquêtes sur les représentations et les pratiques des formateurs ostéopathes du réseau Franc'osteo.

De plus, le graphique 2 suggère que la compétence du formateur consiste principalement à actualiser ses connaissances et à susciter l'intérêt chez les étudiants, afin de faciliter la transmission des connaissances et du concept. L'idée de transmission qui domine expliquerait le choix de la modalité "repérer les difficultés de compréhension ", sélectionnée à plus de $60 \%$. L'étudiant ne doit pas apprendre par lui-même ou avec les autres, mais comprendre ce que le formateur lui explique. La qualité du cours n'arrive qu'en cinquième position et l'animation de groupe en sixième, avec moins d'un quart des réponses, confirmant une faible acculturation à la formation pour adultes. En effet, les représentations des formateurs mettent en avant des compétences liées à la maîtrise des connaissances et des techniques du métier, couplées à l'aptitude à repérer et gérer les incompréhensions individuelles. Selon cette conception, ce n'est ni le collectif, ni les qualités du cours qui sont les principales variables de la réussite des étudiants, mais plutôt les connaissances du formateur. Cette vision correspond davantage à une approche de l'enseignement centrée sur le curriculum, qu'à une approche centrée sur 
l'apprenant et le développement de la personne et de ses compétences, typique de la formation des adultes. Mais l'importance de la modalité «transmettre la philosophie » souligne une relative conscience du caractère humain de cette profession, même si elle n'est du ressort du formateur que pour moins d'un formateur sur deux.

\subsection{L'étudiant idéal : attentif, intéressé et autonome}

Trois qualités à acquérir par les formés émergent. Il s'agit de l'autonomie, de l'esprit critique et de la maturité. Ces qualités font écho à notre question sur la fonction première de l'école d'ostéopathie qui est avant tout de transmettre des techniques et le concept ostéopathique. Lautonomie apparaît dès lors comme un moyen de faciliter l'atteinte des objectifs (apprendre les connaissances transmises) et non comme une finalité développementale (apprendre à gérer et orienter ses apprentissages). Les formateurs attendent des qualités chez les formés qu'ils ne resituent pas au niveau du rôle de l'école. Autonomie, esprit critique et maturité sont des prérequis à la bonne réception des enseignements, à l'autoévaluation de la compréhension des cours, à la conduite de recherches personnelles. Ainsi, l'étudiant doit être un sujet apprenant, réflexif et capable de méta-apprentissages (Tremblay, 2003). Dans ce schéma, le formateur constitue l'une des ressources pour gérer les apprentissages, qu'il appartient aux formés de mobiliser. Le fait de percevoir l'autonomie comme un préalable marque une conception relativement naïve des processus d'apprentissage, qui confirme la faible formation en matière pédagogique. En effet, l'autonomie des individus constitue simultanément une finalité et un moyen de l'apprentissage (Albero \& Bucher-Poteaux, 2010) ; c'est pourquoi il apparaît nécessaire de travailler les méta-compétences, support à l'autoformation en parallèle des disciplines, ce qui implique, de la part des formateurs, une posture et des pratiques spécifiques (AckerKessler, 2015). Par exemple, se considérer comme personne ressource plutôt que transmetteur de savoirs, maîtriser les fonctions d'ingénieur pédagogique, favoriser les situations de questionnement, de recherche et de résolution de problème, avoir recours à l'évaluation formative plutôt que sommative, soutenir l'auto-évaluation tout au long des cinq ans de formation, provoquer l'interdisciplinarité, tendre vers une individualisation de la formation, travailler sur le sens des apprentissages et la motivation, travailler en équipe de formateurs, animer la coopération entre apprenants, etc.

\subsection{La formation des formateurs ostéopathes, une priorité relative}

À 88 \%, les formateurs déclarent se former grâce aux échanges informels entretenus avec leurs pairs, et près de la moitié disent mobiliser leur réseau personnel privé dans ce but. Seul un tiers environ déclare avoir recours à de la formation interne. Les formateurs expriment une préférence pour la formation informelle (Carré \& Charbonnier, 2004), «en contrebande » de l'institution, dirait Jean-François Marcel (2006) en évoquant ces temps interstitiels où se forgent aussi les compétences pour s'adapter à leur activité grâce aux échanges. Ces choix sont également à relier au manque de disponibilité engendré par la double 
activité, mais qui, dans le même temps, indique une hiérarchisation. Les activités de thérapeute passent avant la formation à l'enseignement parce que l'identité d'ostéopathe prévaut (Perez-Roux, 2010), et/ou parce qu'elles sont plus rémunératrices. La formation informelle apparaît donc plus évidente, car plus souple que sa cousine formelle. C'est pourquoi $60 \%$ choisissent le diplôme d'université (DU) pour se former à la pédagogie, plutôt qu'un Master (10\%), plus lourd, ou que les "formations courtes d'une journée " $(5 \%)$. Nous déduisons de ces résultats une vision stratégique de la formation chez les formateurs, devant permettre le développement des compétences, renforcer le parcours professionnel, sans pour autant être invasive. Concernant les contenus de ces formations, aucune thématique relative aux compétences générales de formateur ne ressort. Nous soulignerons surtout le faible intérêt $(20 \%)$ pour les méthodes d'enseignement d'une technique de soin spécifique à l'ostéopathie, alors que cela reste un enjeu majeur et demeure du ressort des compétences incorporées. On peut en conclure que les formateurs ostéopathes n'identifient pas de difficultés sur ce point, alors même que les formés reviennent sur leurs difficultés face aux examens de pratiques. Il en va de même pour "l'encadrement de mémoires " et "les bases de la méthodologie de recherche ", choisis par $20 \%$ seulement de l'échantillon, alors que $70 \%$ des sondés se déclarent "insuffisants " ou " passables » en ces domaines. Cela signifie qu'identification d'une difficulté et tentative de remédiation ne sont pas encore reliées. Enfin, les choix relatifs à la manière idéale de former les collègues délaissent le recours à un conseiller pédagogique, choisi dans $20 \%$ des cas, ou à l'auto-confrontation filmée, choisie par $9 \%$ seulement. Là encore, il semble que les formateurs conçoivent leur développement professionnel surtout comme des temps de mutualisation entre pairs. Cette importance des pairs coïncide avec la place qu'ils tiennent (68\% des réponses) parmi les acteurs les plus aptes à accompagner les formateurs. Les apprentissages relèvent autant de l'expérience professionnelle directe que de la socialisation professionnelle, et sont d'ordres individuel et collectif (Marcel,2006).

Ainsi, la socialisation professionnelle est un processus ni continu, ni linéaire, comprenant des aspects cognitifs, affectifs et interactifs et des moments de discontinuité, de tensions, de contradictions, voire de ruptures (Dubar, 2015). Elle prend forme dans l'interaction entre l'individu et son environnement matériel et social de travail, et produit un rapport à des valeurs, à certaines connaissances du milieu, et à certaines compétences.

\subsection{Formateur ostéopathe : une identité professionnelle à construire}

Une des surprises de ces résultats est l'absence de transposition de la vision du développement professionnel des formateurs à celle des formés. En effet, que ce soit la faible sélection des compétences relatives à la gestion de groupe, la centration sur les contenus ou encore la vision de l'autonomie des étudiants, les formateurs ne semblent pas percevoir le caractère professionnalisant de la formation, ni leur rôle dans les dimensions sociales de cette professionnalisation. 
La posture d'enseignement qui se dessine à travers ces réponses reste celle, très classique, de transmetteur de connaissances, faisant peu de place aux conditions favorisant la construction de l'apprentissage et de l'accompagnement des apprenants dans ce cheminement, même si une réelle appétence à entrer dans un développement professionnel a été exprimée lors de nos rencontres. Cette appétence puise peut-être sa source dans la méconnaissance du métier de formateur, car comme nous le soulignions, ces formateurs sont avant tout des professionnels du soin. Ces éléments entrent en résonance avec nos observations menées lors des entretiens collectifs ou lors de notre quotidien au sein de l'école. Nous n'y avons pas repéré l'utilisation récurrente d'indice culturel propre au métier de formateur d'adultes, tels que la reconnaissance de l'expérience des formés comme ressources de formation, une prise en compte significative de la dynamique de groupe, ou le reflexe du travail en équipe pédagogique qui reste rare et non organisé. Bien que le public encadré soit constitué de jeunes adultes, que la finalité de la formation soit professionnalisante et que l'anticipation de l'usage des apprentissages soit élevée (Boutinet, 1995), la formation actuelle ne tient pas compte des trois caractéristiques de la formation des adultes, à savoir la prise en compte, en une dynamique unique, des parcours individuels, personnels et intellectuels des apprenants, le recours à la diversité des interactions offertes par les technologies de la communication et de l'information et la centralité du principe d'autonomisation dans l'apprentissage (Poisson, dans Carré \& al., 2010). Cette perception non professionnalisante de la formation s'explique par le cadre législatif relatif aux cinq années d'exercice en tant qu'ostéopathe diplômé pour être formateur. Aussi, le fait de n'avoir pas encore investi dans la professionnalisation de leurs salariés à travers la constitution d'espaces collectifs de réflexion sur le travail, la mise à disposition de ressources scientifiques et techniques de la formation ou le montage d'un système de capitalisation et de mutualisation des productions des formateurs, montre que les enjeux de ce processus commencent seulement à être perçus au sein des écoles. Nous confirmons donc notre hypothèse d'un besoin de construire une identité professionnelle de formateur ostéopathe, partiellement explicite (l'identification de leurs besoins de formation). Nous confirmons également l'hypothèse de troubles identitaires, déduite de leurs réponses. Concernant leurs représentations de leur métier d'ostéopathe, les contours de cette identité restent à déterminer, les éléments du monde commun à identifier, et la cohérence et la constance des comportements du collectif des formateurs à renforcer. Pour autant, la volonté marquée de se former entre pairs, évoquée au point précédent, pourrait constituer l'indice d'un sentiment d'appartenance qui témoignerait d'un souhait de constituer un groupe professionnel à partir d'une identité propre. 


\section{Conclusion}

Le besoin de professionnalisation du métier de formateur ostéopathe, pressenti pour expliquer la variabilité des performances entre les groupes de formés, est conforté par notre démarche exploratoire. En effet, les attentes et les représentations des formateurs concernant la formation, leur vision des apprenants et l'absence de diplôme en formation des adultes, confirment la prégnance d'une expérience curriculaire - calée sur l'enseignement scolaire - en partie inappropriée au caractère professionnalisant de la formation. Cette culture entretient une approche disciplinaire disjonctive du métier, qui rend plus délicate, pour les futurs ostéopathes, l'élaboration de modèles opératoires individuels et holistiques du soin intégrant les composantes relationnelles, déontologiques et sensorielles de l'activité.

Enfin, le manque constaté de professionnalisation en termes d'habiletés pédagogiques, ne permet pas actuellement d'interroger collectivement cette tension entre la vision "scolaire » dominante et la finalité professionnalisante du diplôme.

Notre étude suggère aussi l'existence d'une non-intégration entre l'identité du praticien et celle du formateur, favorisant la première au détriment de la seconde. La priorisation des ressources des personnes tend à porter vers le métier d'ostéopathe, plus solidement ancré plutôt que vers celui de formateur ostéopathe, limitant de fait l'implication des personnes dans les espaces dédiés à la dynamique de négociation identitaire, au développement professionnel ou à la définition identitaire collective du métier de formateur-ostéopathe. La promotion de l'implication des professionnels dans ces espaces apparaît donc comme une condition à la professionnalisation des acteurs portant un double métier. Ainsi, l'idée initiale largement partagée dans le milieu des ostéopathes, consistant à dire qu'un bon professionnel en exercice constitue un bon formateur, est à relativiser. Il apparaît plus opportun de trouver un équilibre entre activité professionnelle et activité de formation pour garantir le développement de formateurs-ostéopathes compétents. Ceci suggère la création des conditions d'un rééquilibrage entre les deux composantes de la double activité pour tendre vers une réévaluation de l'identité professionnelle relative aux deux métiers. Un tel rééquilibrage donnerait l'occasion de réinterroger cette tension entre vision scolaire et finalité professionnalisante. Il permettrait enfin d'évaluer l'impact de la vision technocratique de la référentialisation de la formation et de la profession.

Cette étude montre enfin que la création d'un dispositif de formation et de professionnalisation des formateurs ne constitue pas une réponse suffisante aux difficultés observées. Nous avons montré que la professionnalisation des formateurs à un métier dépend de la clarté et du partage de la vision du métier auquel on forme, sans lesquels il est difficile d'élaborer et d'interpréter un référentiel de formation pertinent. Or, dans le cas des écoles d'ostéopathie, cette étude suggère que le flou entourant le concept ostéopathique - qui articule les principes fondateurs de la discipline et guide les praticiens dansl'élaboration de leurs modèles opératoires -, tout commela diversité des conceptions de la profession seraient des effets du processus de professionnalisation de l'ostéopathie encore 
en cours. Il en découle au moins deux courants ciblés par l'enquête, entre lesquels les sondés se positionnent : d'un côté, une approche priorisant l'éthique et la relation humaine, et de l'autre, une approche techniciste davantage centrée sur la thérapie et moins sur la prévention. Il semble donc que la professionnalisation des formateurs ostéopathes reste en partie dépendante du processus de professionnalisation du métier d'ostéopathe qui se situe encore à son troisième stade, celui de la régulation économique et sociale (Dubar $\&$ Tripier, op. cit.) et pour lequel il faut attendre le résultat des négociations qui en découlent à propos de la vision du métier et de son positionnement vis-à-vis de la médecine. Un débat auquel l'analyse de l'activité va contribuer en apportant une connaissance plus fine du métier.

\section{Bibliographie}

Acker-Kessler P. (2015), Posture professionnelle enseignante et développement de l'autonomie dans l'apprentissage des langues : une approche située, thèse en sciences de l'éducation, Université de Strasbourg. En ligne : http://www.theses.fr/2015STRAG056

Aguhlon C. (1998), "Les référentiels de formation comme expression des relations formation-emploi : clarification ou confusion? ", in Les professions de l'éducation et de la formation, Bourdoncle R. \& Demailly L. (Dir.), Villeneuve-d'Ascq, Presses Universitaires du Septentrion, pp. $117-128$

Ardoin T. (2015), "Des professionnalités sous tension : quelles (re)constructions dans les métiers de l'humain?", in Bodergat J.-Y. \& Buznic-Bourgeacq P. (Dir.), Louvain-laNeuve, De Boeck, pp. 41-61.

Albero B. \& Bucher-Poteaux N. (2010), Enjeux et dilemmes de l'autonomie : une expérience d'autoformation à l'université : étude de cas, Paris, Édition de la Maison des sciences de l'homme.

Balas S. (2011), Le référentiel, un outil de formation, un instrument de développement du métier : le métier de masseur-kinésithérapeute en référence, thèse en formation des adultes, Paris, CNAM. En ligne : http://www.theses.fr/2011CNAM0806

Balas S. (2012), "Transmettre un métier ? L'exemple des masseurs-kinésithérapeutes ", communication présentée à la Biennale internationale de l'éducation, de la formation et des pratiques professionnelles. En ligne : https://halshs.archives-ouvertes.fr/ halshs-00790594

Bardin L. (2013), L'analyse de contenu, Paris, Presses Universitaires de France.

Berger J.-L. \& D’Ascoli Y. (2011), « Les motivations à devenir enseignant : revue de la question chez les enseignants de première et deuxième carrière », Revue française de pédagogie. Recherches en éducation, $\mathrm{n}^{\circ}$ 175, pp. 113-146. En ligne : https://doi. org/10.4000/rfp.3113 
Boutinet J.-P. (1995), Psychologie de la vie adulte, Paris, Presses Universitaires de France.

Bodergat J.-Y. \& Buznic-Bourgeacq P. (2015), Des professionnalités sous tension : quelles (re)constructions dans les métiers de l'humain?, Louvain-la-Neuve, De Boeck.

Carré P. \& Charbonnier O. (2004), Les apprentissages professionnels informels, Paris, L'Harmattan.

Coues E., Hoffman H. \& Comeaux Z. (2012), L'âme de l'ostéopathie : réflexions classiques et contemporaines sur le sens de la vie et leur intérêt pour la santé, Paris, Éditions Frison-Roche.

David A. (1999), "Logique, épistémologie et méthodologie en sciences de gestion ", VIIIème Conférence Internationale de l'A.I.M.S, Châtenay Malabry. En ligne sur : https://www.strategie-aims.com/events/conferences/15-viiieme-conference-de-l-aims/ communications/2334-logique-epistemologie-et-methodologie-en-sciences-de-gestion

De Ketele J.-M. \& Maroy C. (2006), "Quels critères de qualité pour les recherches en éducation ? ", in Paquay L., Crahay M. \& De Ketele J.-M (Dir.), L'analyse qualitative en éducation. Des pratiques de recherche aux critères de qualité. Hommage à Huberman, Bruxelles, De Boeck.

Desjours C. (2001), «Subjectivité, travail et action », La Pensée, n 328, pp. 7-20.

Dubar C. (2015), La socialisation : construction des identités sociales et professionnelles, Paris, Armand Colin.

Dubar C. \& Tripier P. (1998), Sociologie des professions, Paris, Armand Colin.

Durat L. \& Mohib N. (2008), «Le développement des compétences au regard de l'engagement dans l'agir professionnel ", Questions Vives, n 10, pp. 25-41.

Escofier B. \& Pagès J. (2008), Analyses factorielles simples et multiples : objectifs, méthodes et interprétation, Paris, Dunod.

Fray A.-M. \& Picouleau S. (2010), « Le diagnostic de l'identité professionnelle : une dimension essentielle pour la qualité au travail », Management Avenir n ${ }^{\circ} 38$, vol. 8, pp. 72-88. En ligne : https:/www.cairn.info/revue-management-et-avenir-2010-8page-72.htm

Garnier F. \& Braccini V. (2017), « Enquête préparatoire à la création d'un référentiel de compétences propre au métier de formateur en ostéopathie ", La Revue de l'Ostéopathie $\mathrm{n}^{\circ} 18$, pp. 29-36.

Gueullette J.-M. (2014), L’ostéopathie, une autre médecine, Rennes, Presses Universitaires de Rennes.

Issartel L. \& Issartel M. (1983), L'Ostéopathie exactement, Paris, R. Laffont.

Le Corre F. \& Toffaloni S. (2007) L'ostéopathie, Paris, Presses Universitaires de France. 
Liu M. (2013), La dynamique des organisations : l'émergence des formes démocratiques, Paris, L'Harmattan.

Marcel J.-F. (2006), " Le Collectif d'enseignants. Explorations théoriques et empiriques d'un nouvel acteur des systèmes éducatifs ", L'enseignement aujourd'hui : contextes sociopolitiques, formation et pratiques d'enseignement en question, $\mathrm{n}^{\circ}$ 5, pp. 88-99.

Mayen P. (2018), «S'écarter du travail pour mieux l'apprendre. Une réflexion pour l'ingénierie de formation en situation de travail et pour la conception d'organisations apprenantes ", Éducation permanente, n 216, pp. 141-158.

Parent F. (2014), "Seuls les médecins se piquent d'acupuncture? ", Terrains travaux $\mathrm{n}^{\circ} 25$, pp. 21-38.

Parent F. (2017), Gouverner le bien être: travail des normes et mobilisations autour des pratiques professionnelles de la médecine chinoise en France, thèse en science politique, Université de Toulouse 1. En ligne : http://www.theses.fr/165732822

Parent F. (2008), «Chapitre 8. Formation, compétences et constructivisme : le référentiel d'évaluation, outil de cohérence dans les programmes de formation en santé ", Évaluer pour former, Louvain-la-Neuve, De Boeck, pp 111-139. En ligne : http://www.cairn. info/article.php?ID_ARTICLE=DBU_BAILL_2008_01_0111

Parent Fl. \& Jouquan J. (2013), Penser la formation des professionnels de la santé : une perspective intégrative, Bruxelles, De Boeck.

Parent Fl. \& Jouquan J. (2015), Comment élaborer et analyser un référentiel de compétences en santé ?, Louvain-la-Neuve, De Boeck.

Pastré P. (1997), "Didactique professionnelle et développement ", Psychologie française, $\mathrm{n}^{\circ} 1$, (42), pp. 89-100.

Perez-Roux T. (2010), "Processus de professionnalisation et dynamiques identitaires : deux études de cas chez les enseignants de lycée professionnel en France ", Nouveaux cahiers de la recherche en éducation, $\mathrm{n}^{\circ} 13$, (1), pp. 83-101. En ligne : https://doi. org/10.7202/1017462ar

Poisson D. (2010), "Les dispositifs d'autoformation et leurs ingénieries »?, in Carré P., Moisan A. \& Poisson D., L'autoformation : perspectives de recherche, Paris, Presses Universitaires de France.

Poteaux N. (2007), L'innovation pédagogique entre recherche et terrain, habilitation à diriger des recherches, Strasbourg, Université Louis Pasteur.

Saint-Jean M., Isus Barado S., Paris Manas G. \& Mace A. (2014), « La recherche-intervention comme accompagnement du changement : le cas d'une formation de formateurs ", Les Dossiers des Sciences de l'Éducation, n 31, pp. 31-48. En ligne : https://doi. org/10.4000/dse. 558 
Tardif J. (2012), Devenir ostéopathe. Agir avec compétence, St-Étienne, SNESO.

Tremblay N.-A. (2003), L'autoformation : pour apprendre autrement, Montréal, Les Presses de l'Université de Montréal.

Vidal-Gomel C. \& Rogalski J. (2007), « La conceptualisation et la place des concepts pragmatiques dans l'activité professionnelle et le développement des compétences ", in Activités 04, n 4-1. En ligne : https://doi.org/10.4000/activites.1401

Wittorski R. (2009), "À propos de la professionnalisation », Encyclopédie de la formation, in Barbier J.-M., Bourgeois E., Chapelle G. \& Ruano-Borbalan J.-C. (Dir.), Paris, Presses universitaires de France, pp. 781-93.

Wittorski R. (2015), «Questions posées à la professionnalisation aux métiers de l'humain ", Des professionnalités sous tension : quelles (re)constructions dans les métiers de l'humain ?, in Bodergat J.-Y. \& Buznic-Bourgeacq P. (Dir), Louvain-la-Neuve, De Boeck, pp. 31-42. 